\title{
ON BOTH SPATIAL AND VELOCITY DISTRIBUTION OF SPUTTERED PARTICLES IN MAGNETRON DISCHARGE
}

\author{
C. Vitelaru, V. Pohoata, V. Tiron, C. Costin, G. Popa \\ “Alexandru Ioan Cuza" University, Department of Physics, 700506 Iasi, Romania
}

\section{Article Info \\ Received: 28 December 2011 \\ Accepted: 19 January 2012}

Keywords: pulsed magnetron, tunable diode laser spectroscopy, neutral distributions

\begin{abstract}
The kinetics of the sputtered atoms from the metallic target as well as the time-space distribution of the argon metastable atoms have been investigated for DC and high power pulse magnetron discharge by means of Tunable Diode - Laser Absorption Spectroscopy (TD-LAS) and Tunable Diode - Laser Induced Fluorescence (TD-LIF). The discharge was operated in argon (5-30 mTorr) with two different targets, tungsten and aluminum, for pulses of 1 to $20 \mu \mathrm{s}$, at frequencies of 0.2 to $1 \mathrm{kHz}$. Peak current intensity of $\sim 100 \mathrm{~A}$ has been attained at cathode peak voltage of $\sim 1 \mathrm{kV}$. The mean velocity distribution functions and particle fluxes of the sputtered metal atoms, in parallel and perpendicular direction to the target, have been obtained and compared for DC and pulse mode.
\end{abstract}

\section{Introduction}

Nowadays magnetron discharge is extensively used mainly for deposition of both metallic and compound thin films for large varieties of applications in microelectronics, hard coatings, optical layers with desired physical properties $[1,2]$. These very good performances in industrial applications did not stop but, on contrary, asked for more investigation for elementary processes understanding starting with sputtering phenomena, transport of the sputtered atomic material within the plasma volume and its deposition on the substrate. Increasing performances brought by high power impulse magnetron sputtering (HiPIMS) [3, 4] to the quality of the deposited layer and its adherence to the substrate, led to an increased interest in developing of new techniques and methods for detailed characterization of the sputtered particles kinetic.

Moreover, since HiPIMS are currently operated at power density on the cathode surface comparable with power density encountered in fusion technology at the divertor surface of 
JET or ITER devices [5], HiPIMS brought an additional interest in the field of fundamental understanding of sputtering process under high ion fluxes. Fusion program needs more information on the properties of the materials used in designing and building-up of the high power fusion reactor. Consequently, special and rather expensive devices, such as Pilot-PSI and MAGNUM-PSI in The Netherlands, JULE-PSI in Germany and VISION I in Belgium[6], were designed and constructed (or are under construction), allowing the investigation of various phenomena, as interaction of high density hydrogen thermal plasma jet with targets made of tungsten, carbon or other materials used in fusion technology.

These devices were designed for rather long duration plasma - target interaction, longer than a second, comparing with duration of the HiPIMS system where pulse duration is usually shorter, typically tens or hundreds of microseconds [7]. However, various methods and investigation techniques (with good space- and time-resolution) used for the study of plasma and sputtered material kinetics as well as the rather low cost HiPIMS recommend such a system for the study of the phenomena taking place at the interaction of the high density plasma with metallic targets at rather short duration time, which might correspond to plasma wall interaction in fusion devices during instabilities or transient phenomenon as e.g. ELMs [8].

The aim of this paper is the presentation of the experimental results on characterization of the sputtered particles kinetic from a metallic target in a magnetron discharge operated at high power pulse in argon for pulse duration time less than $10 \mu \mathrm{s}$. The target material was tungsten, carbon or aluminum. In magnetron discharges, the native energy distribution of particles ejected by sputtering process depends on the gas pressure being modified by collisions with buffer gas atoms, and influenced by the current density profile, which is strongly related to the non-uniform magnetic field configuration. Consequently, the energy distribution function of the sputtered atoms in the gas phase can be very complex, changing drastically both spatially and temporarily from the target to the substrate, of which characterization is asking special diagnostic technique. Moreover, mean kinetic energy or "temperature" of both sputtered particles and buffer gas atoms in front of the target, as well as their density are strongly dependent on the position in front of the target and the moment during the pulse discharge.

Fortunately, the Tunable Diode - Laser Absorption Spectroscopy (TD-LAS) and Tunable Diode - Laser Induced Fluorescence (TD-LIF) allow investigation of the both local density and the energy distribution of the absorbing atoms [9]. Indeed, the narrowness of laser diode wavelength profile and the high spatial resolution coupled with axial symmetry of the 
circular magnetron allow the TD-LAS technique to be used for measuring absolute values of the both density and temperature of absorbing species. Moreover, it was pointed out that the narrowness of laser diode wavelength profiles and the high spatial resolution permit the deconvolution of TD-LIF signal into two, or more, well defined distribution functions, corresponding to thermalized or quasi-thermalized and energetic sputtered particles, respectively.

Consequently, these two techniques were used to measure either the velocity distribution functions in two perpendicular velocity directions, namely normal $\left(v_{y}\right)$ and parallel to the target $\left(v_{r}\right)$ as well as the corresponding flux distributions. It is also possible to evaluate the distance from the target where the sputtered energetic particles are thermalized. Moreover, in order to have a better description of the particles within the plasma volume in front of the target the density of the argon metastable and temperature of the buffer gas were also measured.

These methods primarily used for DC steady state magnetron discharge [9-11] were also developed and used for investigation of the specified kinetic parameters within pulsed system as time resolved - direct absorption profile (TR-DAP)[12].

\section{Experimental set-up and principle}

The experimental arrangement for both techniques, TD-LAS and TD-LIF, respectively, used for kinetic characterization of the particles in the region of plasma-solid target interaction is presented in Fig. 1. The plasma source is a planar circular magnetron of 56 $\mathrm{mm}$ diameter placed into a cylindrical stainless steel reactor chamber of $15 \mathrm{~cm}$ in diameter and $35 \mathrm{~cm}$ in height. The laser beam can cross the plasma volume either parallel to the target surface, or normal to it. The chamber was pump-down by a turbo molecular system to a base pressure of about $10^{-6}$ Torr. The Argon was used as working gas at the pressure in the range of 5 to 30 mTorr.

The magnetron was either DC supplied (voltage up to $400 \mathrm{~V}$ and discharge current intensity up to $0.4 \mathrm{~A}$ ) or by a high power adapted pulsed generator [13]. Typical pulse shape of the applied magnetron voltage and current intensity is presented in Fig.2 and it has the following characteristics: the pulse duration between $1-20 \mu \mathrm{s}$, the frequency $0.2-1 \mathrm{kHz}$ and the pre-ionization current intensity of $4 \mathrm{~mA}$. This minimum current is flowing through the discharge between the power pulses and discharge voltages are self-adjusted according to experimental conditions. The cathode peak voltage can reach $-1 \mathrm{kV}$ and the peak current intensity may reach even $100 \mathrm{~A}$. The short duration pulses used in the experiment correspond 
only to the initial stage of longer duration pulses, such as the ones used by Anders et al. ${ }^{7}$ so that it is very probable that self-sputtering process of the target metal is not yet dominant in these experiments.

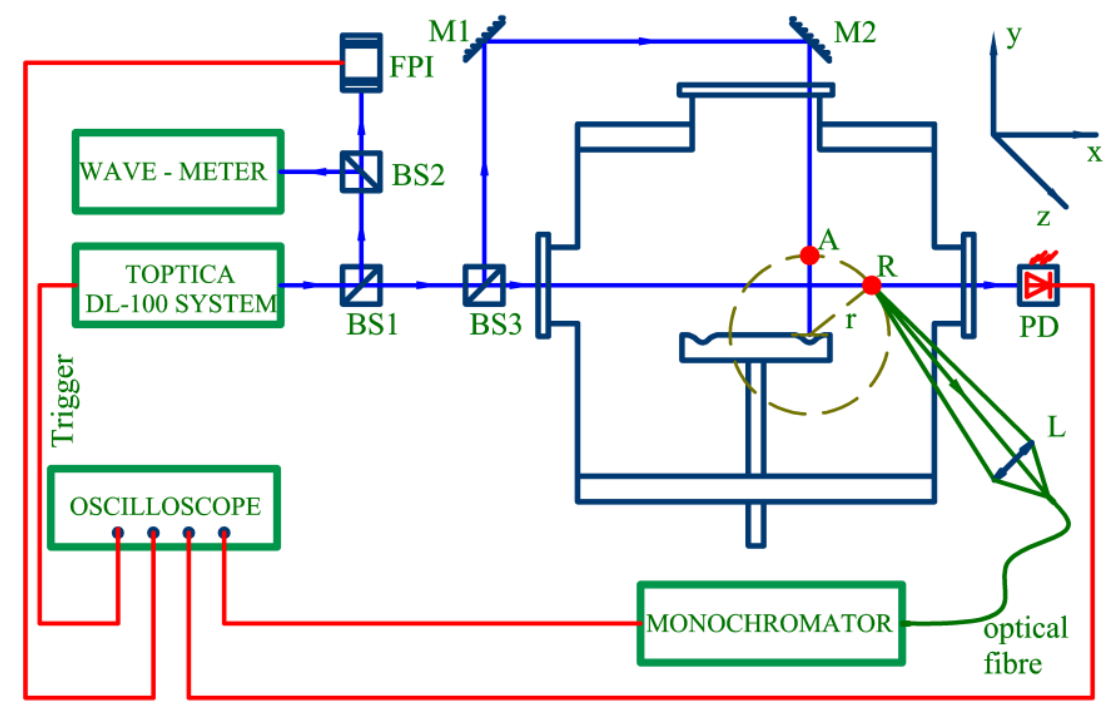

Fig. 1. Schematic view of the experimental set-up: FPI - Fabry-Perot interferometer, BS1, BS2, BS3 beam-splitters, M1, M2 - mirrors, PD - photodiode detector, L - collimating lens; A and R mark two investigated small volumes, equidistantly situated at $20 \mathrm{~mm}$ from the magnetron race track.

The laser source used in plasma diagnostic in both cases TD-LAS and TD-LIF was a Toptica Photonics DL 100 system with tunable single-mode blue-laser diodes for metal atoms and red-laser diode for argon metastables, each centered at the resonance wavelength $\lambda_{0}$ corresponding to the investigated atom. To improve the narrowness of laser emission line for fine spectroscopic measurements and select single mode operation, the diode system is commonly mounted in an external laser cavity driven by piezoelectric actuator. Tuning the cavity, single mode operation is possible for a typical wavelength scanning range of 2 to $5 \mathrm{pm}$ for near-UV and $20 \mathrm{pm}$ for the red range, without laser mode hop. The scan control module (SC 110-Toptica) enables the laser wavelength scanning with a frequency between $0.01 \mathrm{~Hz}$ and $6 \mathrm{kHz}$. Typically $5 \mathrm{~Hz}$ was used. Nevertheless, the system stability is achieved by combining the actuator scan with the diode current intensity controller within a feedback loop. The laser wavelength is controlled by the diode current intensity, on one hand, and by the angle of diffraction grating mounted in the external cavity, on the other hand. The scanning range available without cavity mode jump was typically $10 \mathrm{pm}$ obtained by controlling the diode scan current and constant diode temperature. The laser wavelengths correspond to the metal atom transitions from the ground state to excited levels as following: i) aluminum, absorption at $\lambda_{0}=394.512 \mathrm{~nm}$ for transition $3 \mathrm{~s}^{2} 3 \mathrm{p}(1 / 2) \rightarrow 3 \mathrm{~s}^{2} 4 \mathrm{~s}(1 / 2)$ and ii) tungsten, 
absorption at $\lambda_{0}=407,436 \mathrm{~nm}$ for transition $5 \mathrm{~d}^{5}\left({ }^{6} \mathrm{~S}\right) 6 \mathrm{~s} \rightarrow 5 \mathrm{~d}^{5}\left({ }^{6} \mathrm{~S}\right) 6 \mathrm{p}$. For Argon atom the absorption at $\lambda_{0}=811.531 \mathrm{~nm}$ for transition of a metastable level $4 s^{2}[3 / 2]_{2} \leftrightarrow 4 p^{2}[5 / 2]_{3}$. The TD-LIF technique signal was detected as the transition from these latter excited levels to a lower ones as following: for aluminum the wavelength $\lambda=396.264 \mathrm{~nm}$ for transition $3 \mathrm{~s}^{2}$ $\left.4 \mathrm{~s}(1 / 2) \rightarrow 3 \mathrm{~s}^{2} 3 \mathrm{p}(3 / 2)\right)$ and for tungsten due to very high emission coefficient $\left(A_{k i}=10^{7} \mathrm{~s}^{-1}\right)$ the fluorescent transition was measured for the same wavelength $\lambda=407.436 \mathrm{~nm}$ and transition $5 d^{5}\left({ }^{6} S\right) 6 s \rightarrow 5 d^{5}\left({ }^{6} S\right) 6 p$.



Fig.2. Typical current and voltage shapes in HiPIMS discharge with $\mathrm{W}$ target, $p=30$ mTorr.

Using an external optical arrangement consisting of high reflectivity mirrors and beam splitters (Fig.1), the laser beam was separated into two beams: one probes the plasma volume and it is partly absorbed by corresponding atoms, while the other one goes through a FabryPerot interferometer for the wavelength scale calibration $\left(\Delta \lambda=\lambda_{\text {laser }}-\lambda_{0}\right)$. Thus the wavelength scale is transformed to velocity scale using the Doppler shift formula $v=c \Delta \lambda / \lambda_{0}$ and the TD-LIF signal is then represented as a velocity distribution function $(v d f)$ of the absorbing species. Prior to the measurements, the linearity between TD-LIF signal and laser power was checked. A further normalization was employed in order to consider the laser power variation in the laser wavelength scanning range for each specific laser diode.

The width of the single mode laser line $\left(5 \times 10^{-6} \mathrm{~nm}\right)$ is much lower than the Doppler width $\left(\sim 8 \times 10^{-4} \mathrm{~nm}\right)$ of the absorbing metal line at room temperature. Consequently, due to this high spectral accuracy, the Doppler broadening can be easily resolved using the TD-LIF technique. Each of the two laser beams probes alternately the $v d f$ in the direction parallel to the target ('radial laser beam' or $x$ direction in Fig.3) and perpendicular to it ('axial laser 
beam' or $y$ direction in Fig.3). The schematic view reported in Fig.3 shows the relative position of the sputtered target surface and the laser beam. Moreover, using a movable detection optics assembly, consisting of a focusing lens and optical fiber, detection of the fluorescence signal can be obtained all along the laser path in front of the target. The detection signal for the TD-LAS measurement includes the information of the total plasma volume probed by laser beam along its path, while the LIF signal may pick-up the signal from different elementary volumes, located along the laser beam. Moreover, in the latter case the optical signal was then passed through a monochromator in order to select the fluorescence wavelength specific to the chemical element investigated ( $\mathrm{Al}$ or $\mathrm{W}$ ) then transformed into an electrical signal by a photomultiplier and registered on a digital oscilloscope (as in Fig.1).

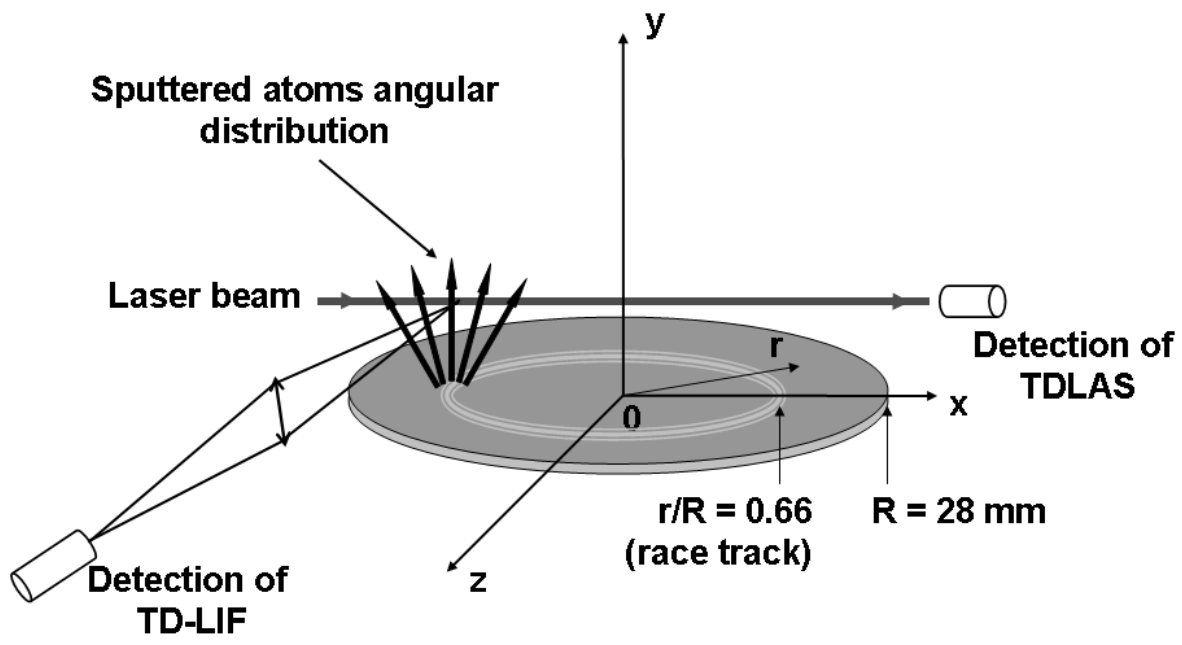

Fig. 3. The principle of the experimental device for detection of both signals provided by absorption (TDLAS) and laser induced fluorescence (TD-LIF) techniques.

By synchronizing the HiPIMS pulse frequency with the scanning frequency of the diode laser the absorption profile virtually in any instant on the pulse period has been obtain. For $200 \mathrm{~Hz}$ repetition rate of the pulsed power supply, during a laser period $(\sim 0.5 \mathrm{~s})$ about 100 discharge pulses occur. During a post-discharge period $(5 \mathrm{~ms})$ the wavelength variation is less as $0.08 \mathrm{pm}$ so that laser wavelength could be considered as constant. Consequently, we may assume that to each peak corresponds a given absorption wavelength and the period during the peaks corresponds to the time evolution of the absorbance at the specific wavelength. Due to the relatively low gas pressure and plasma density one may also admit that the main broadening process of the spectral lines in the discharge is Doppler one so that information about the thermal energy of the particles can be obtained. 


\section{Experimental results and discussions}

For the sake of clearness concerning the capability of the methods and techniques used for kinetic characterization of the sputtered particles, in this very first part of the chapter the typical results obtained in DC mode of operation of the magnetron discharge are presented. Thus, the typical velocity distribution in the radial direction is shown in Fig.4. It was obtained in DC mode of magnetron discharge operation for aluminum target. The LIF signal was registered in different positions along a diameter of the magnetron target, for a distance of $1 \mathrm{~mm}$ from the cathode surface. More details about this classical system of operation have been already presented [9-11]. As it was already pointed out, the velocity distribution of sputtered metal species measured in the radial direction at low pressure, typically less than 10 mTorr, has twocomponent shape [9]. The corresponding distribution is described as the sum of a symmetrical component representing the quasi-thermalized atoms and of the shifted component representing the energetic atoms which has its maximum around $1 \mathrm{~km} / \mathrm{s}$ in the case of aluminum atoms. Moreover, it has to be noted that the sputtered atoms leave the target with high energy mainly perpendicular to the target but, still bring their contribution to the 'quasi-thermalized' component of the velocity distribution presented in Fig.4.

The experimental results obtained in the perpendicular direction to the cathode surface (not shown here) describe well the anisotropy of the sputtered metal atom kinematics. In this case the laser beam is injected perpendicular to the target surface probing the velocity component perpendicular (normal) to the target. The measurement made in $y$ direction perpendicular to target surface shows clear dependence of the velocity and energy distribution of the sputtered atoms on the distance from the cathode surface, on discharge current intensity and the gas pressure [9-11].

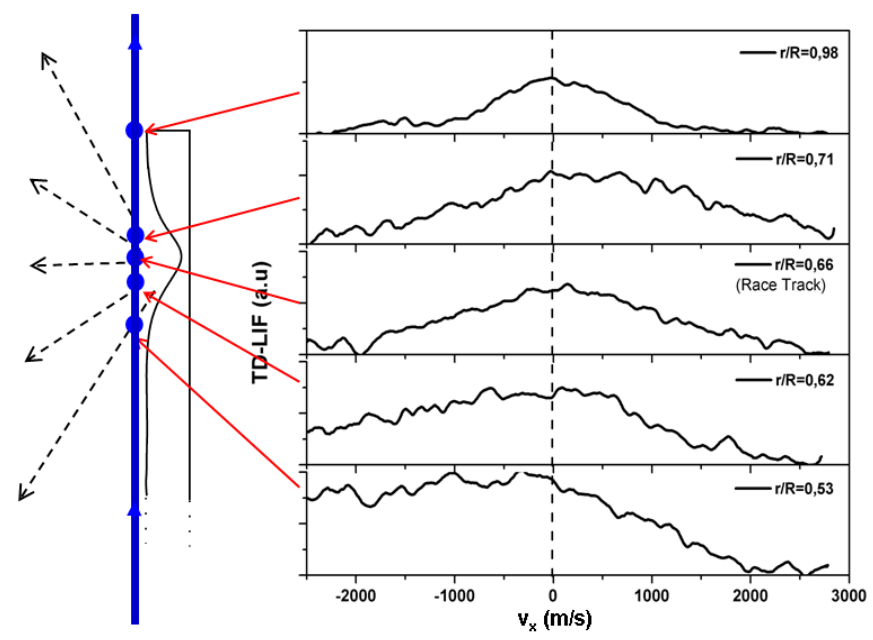

Fig. 4. The $v d f$ radial component for aluminum target taken at $1 \mathrm{~mm}$ distance from the cathode surface, $p=5$ mTorr, $I=0.1$ A (DC mode). 
Simultaneously to TD-LIF measurement, TD-LAS measurement can be performed (Fig.3). Such measurement has been performed using, for instance, the absorption measurements at $394.40 \mathrm{~nm}$ and $396.15 \mathrm{~nm}$ in order to determine the temperature and density of thermalized aluminum atoms in hollow cathode discharge [14], in DC magnetron discharges [15] and even for the time averaged density and temperature of aluminum atoms for power pulsed magnetron sputtering [16]. The TD-LAS technique gives the absorption spectral line profile. When the laser radiation absorption is due to atoms controlled by thermal motion, the absorption profile fits with Gaussian formula which describes a Doppler broadened profile. From this profile one can extract the amplitude of the absorbance profile $k\left(\lambda=\lambda_{0}\right)$ and the full width at half maximum $\left(\Delta \lambda_{\text {FWHM }}\right)$. The density of e.g. aluminum atoms [Al] as absorbing species can be calculated using the following formula, where $f$ is the oscillator strength of the transition:

$$
\left[A l\left(\mathrm{~cm}^{-3}\right)\right]=\frac{k\left(\lambda_{0}\right)\left(\mathrm{cm}^{-1}\right) \Delta \lambda_{F W H M}(\mathrm{~cm})}{8.25 \cdot 10^{-13} \cdot \lambda_{0}^{2}\left(\mathrm{~cm}^{2}\right) \cdot f} .
$$

For the operation in high power pulse mode of the planar circular magnetron described above, the TD-LIF measurements (mean values) were performed for tungsten targets and compared with the DC operation results. The high power pulse mode was realized using the adapted pulse power generator described in section 2, which produces power pulses typically presented in Fig.2. The corresponding current-voltage (I-V) characteristics of the high power pulse discharge for tungsten (W) and carbon (C) targets are presented in Fig.5 for $8 \mu$ s pulse duration.

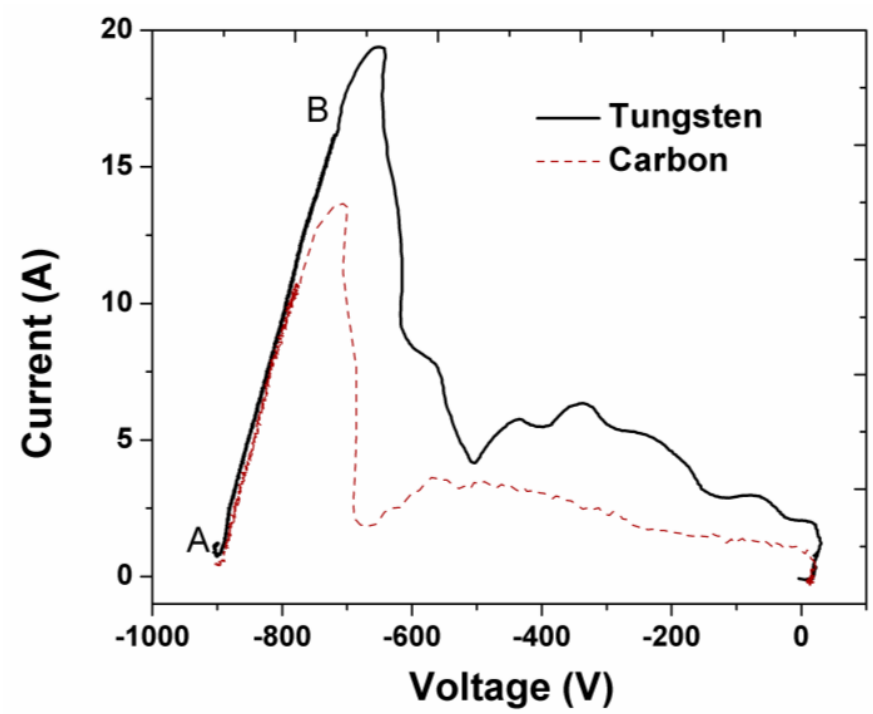

Fig. 5. Current-voltage characteristics of the pulse magnetron discharge for $\mathrm{W}$ and $\mathrm{C}$ targets, $p=10$ mTorr, pulse duration $8 \mu \mathrm{s}$. 
The main part of the pulse ( $92 \%$ from total duration), which corresponds to the $\mathrm{AB}$ region pointed out in Fig.2, shows a clear negative and constant electrical resistance of about $11.7 \Omega$ for tungsten and $11.3 \Omega$ for carbon target. The fact that the electrical resistance is almost independent on the target material (also verified for $\mathrm{Al}, \mathrm{Zn}, \mathrm{Fe}$, Ti targets but not shown here) indicates that the self sputtering process of the target metal is not dominant for the short pulses (less than $10 \mu \mathrm{s}$ ) used in the present experiments. This corresponds to strong increase of the charge carrier production due to strong target sputtering and ionization of the sputtered atoms which have lower ionization energy than argon buffer gas. The maximum power density on the cathode surface is of the order of $10 \mathrm{MW} / \mathrm{m}^{2}$. The non-linear part of the I-V characteristics shows mainly positive impedance, which corresponds to very fast transitory part of the discharge at set-on and set-off of the power pulse, and which last less than $8 \%$ from the total duration of the pulse.

The TD-LIF described technique was used to measure the time averaged $v d f$ of metallic atoms sputtered within the HiPIMS presented above and compared with the similar results obtained in the DC discharge at the same average power. The average includes supposedly large number of metallic atoms sputtered during the $8 \mu$ s power pulse and the number of atoms within afterglow plasma and pre-ionization regime, respectively; when very low plasma density lasts for about 1 ms. Typical results are presented in Fig. 6 for tungsten target with the DC power as parameter. The $v d f$ were measured in two different positions (A and R, specified in Fig.1), each at $20 \mathrm{~mm}$ distance from the race track. These points were selected for information about sputtered atoms in axial (point A, direction oy) and radial (point R, horizontal direction $o x$ ) directions, respectively.

However, in magnetron discharge applications the sputtered particles flux at different positions within the sputtering system is an important quantity, which is directly related to deposition rate of the sputtered material on a substrate. In this view using results as those presented in Fig.6 the corresponding fluxes of the sputtered atoms were also investigated, and compared between the DC and HiPIMS discharge at equivalent average powers. Consequently, the tungsten atom fluxes were calculated in the two points A and R from Fig. 1 and presented in Fig.7 and Fig.8, respectively for two different gas pressure. It is obvious that the time averaged $v d f$ of the sputtered metallic atoms is similar to that obtained in DC regime showing two groups of atoms. One group with almost thermal distribution which correspond to thermal atoms and the second one showing rather large velocity distribution, which is even larger in radial direction than in axial one. 

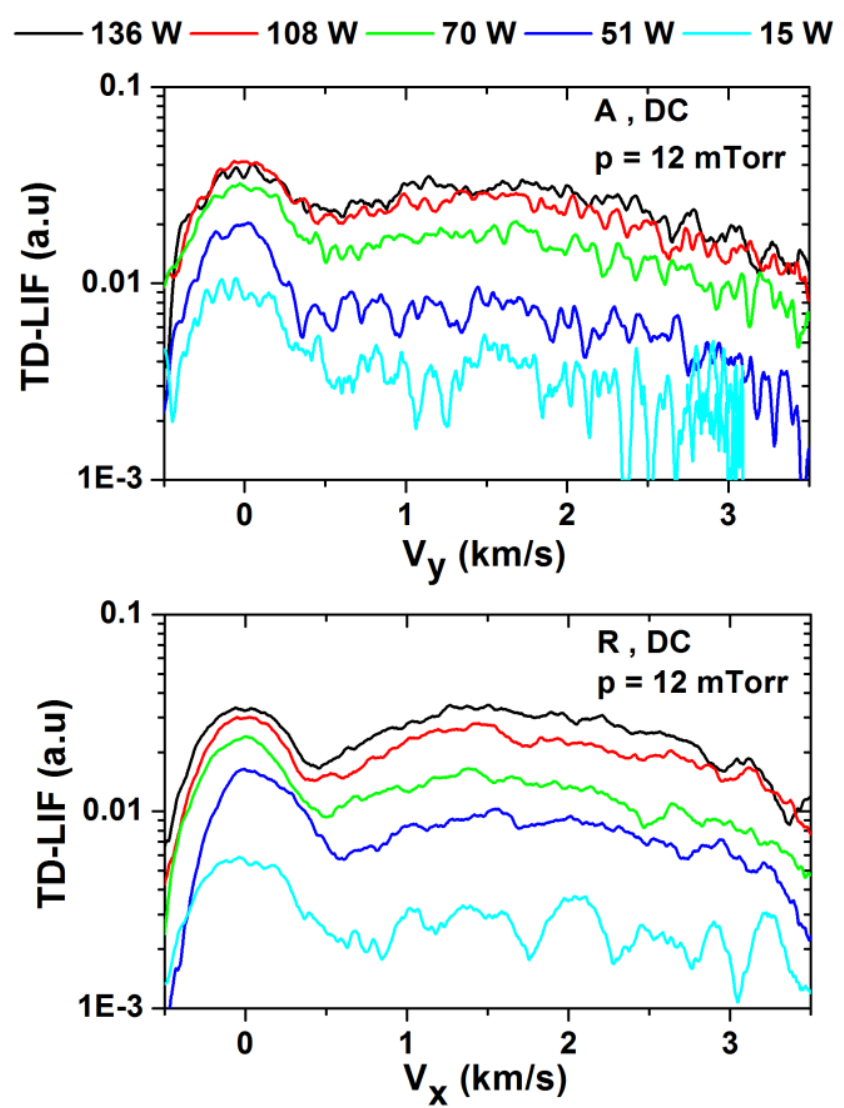

Fig. 6. The dependence of axial $(y)$ and radial $(x)$ velocity distribution function of neutral tungsten atoms, measured at $20 \mathrm{~mm}$ from the race track (labeled as A and R region in Fig.1.) at constant pressure of $12 \mathrm{mTorr}$, as function of the DC discharge power.



Fig. 7. Axial velocity distribution function and neutral tungsten atoms flux, measured at $20 \mathrm{~mm}$ from the race track (labeled as A region in Fig.1.) at constant discharge power of $60 \mathrm{~W}$, in the case of both, DC and HiPIMS discharges, for two pressures. 


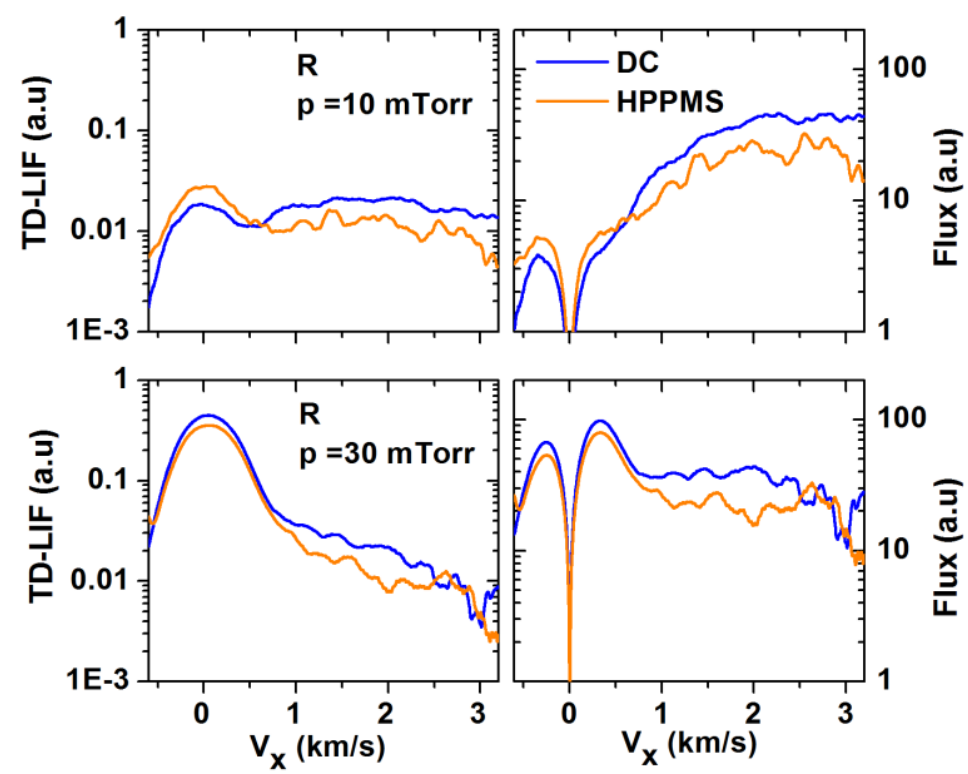

Fig. 8. Radial velocity distribution function and neutral tungsten atoms flux, measured at $20 \mathrm{~mm}$ from the race track (labeled as $\mathrm{R}$ region in Fig. 1.) at constant discharge power of $60 \mathrm{~W}$, in the case of both, DC and HiPIMS discharges, for two pressures.

The results show the following characteristics:

i) In all cases both the $v d f$ and more pronounced corresponding fluxes of the sputtered atoms have diminished values for the HiPIMS operation comparing with the DC one for the same mean power. This result clearly shows that during the high power pulse an important fraction of the sputtered atoms are ionized and the mean value of both atom densities and fluxes of tungsten atoms (which are measured by the TD-LIF technique) is decreased comparing with their value obtained in DC regime of sputtering for the same mean power transferred to the system.

ii) Increasing of the gas pressure has a strong influence on the flux of the sputtered atoms in direction perpendicular to the target. Increasing of the gas pressure leads to increasing of the collision frequency of the sputtered atoms with argon atoms and both number density and fluxes of the fast component of the sputtered atoms are diminished.

iii) The effect of the gas pressure is better emphasized by thermal component of the sputtered atoms within the $v d f$ distribution, which shows strong increase for both axial (A) and radial $(\mathrm{R})$ direction with respect to fast component of the sputtered atoms.

As it was presented in section 2, synchronizing the HiPIMS pulse frequency with the scanning frequency of the diode laser the absorption profile of the laser radiation, at any instant, on the pulse period might be obtained. Moreover, due to the relatively low gas pressure and plasma density one may assume that the main broadening process of the spectral 
lines, constructed as envelop of the series of individuals peaks registered during laser period, is a Doppler one. Information about the thermal energy of the particle system and their number of the both buffer gas atoms (argon) and sputtered atoms (aluminum or tungsten) can be obtained from the reconstructed absorption spectral line due to plasma volume probed by laser beam[12,17] also within the HiPIMS system. In the following the experimental results enlightening some aspects on both transport phenomena of sputtered species from metallic target surface towards substrate and balance between creation and loss process for argon metastables mainly on the time period between the two pulses of a high power pulse magnetron discharge. Comparison between time and space evolution of metallic and argon metastable species $\left(\mathrm{Ar}^{\mathrm{M}}\right)$ shows that the metal atoms are governed by transport phenomena, while the $\mathrm{Ar}^{\mathrm{M}}$ are strongly influenced by electron density variation in time and space.

Typical result on the temporal evolution of the maximum absorption coefficient of the aluminum spectral line is presented in Fig.9 for different distances from the target. It shows that number of aluminum sputtered atoms, probed by the laser beam close to the target surface $(y=1 \mathrm{~mm})$, decrease rapidly in time due to physical processes present in afterglow plasma. Among these processes the transport in both radial and axial directions due to both density gradient and fast sputtered atoms transport from target towards the walls has to be considered. With increasing distance from the target surface $(y=6$ to $36 \mathrm{~mm}$ ) the number of aluminum sputtered atoms, probed by the laser beam in radial section shows first an increasing up to a maximum value followed by a decreasing rate in time. Both maximum values and decreasing rate of the aluminum atoms number decrease with increasing distance from the target surface. However, after $2 \mathrm{~ms}$ the aluminum atoms are still present at a distance of $40 \mathrm{~mm}$ from the cathode surface but no aluminum atoms are detectable in the plasma volume after $5 \mathrm{~ms}$, before the next power pulse.

These results provide information about kinetic of the aluminum sputtered atoms from the circular race track and their spreading in radial - axial directions with larger time spread interval for larger distance from the target.

The metastable argon atoms are also studied by TD-LAS technique during the afterglow and pre-ionization regime between the high power pulses of the magnetron discharge with aluminum target. The presence of the argon atoms all over the discharge volume leads to a different time evolution of the spatial distribution of the argon metastable atoms within afterglow plasma. The first observation is that argon metastable atoms are almost all the time present within the plasma volume due to both afterglow plasma and pre-ionization regime at constant discharge current intensity (4 mA) (see Fig.10a). 


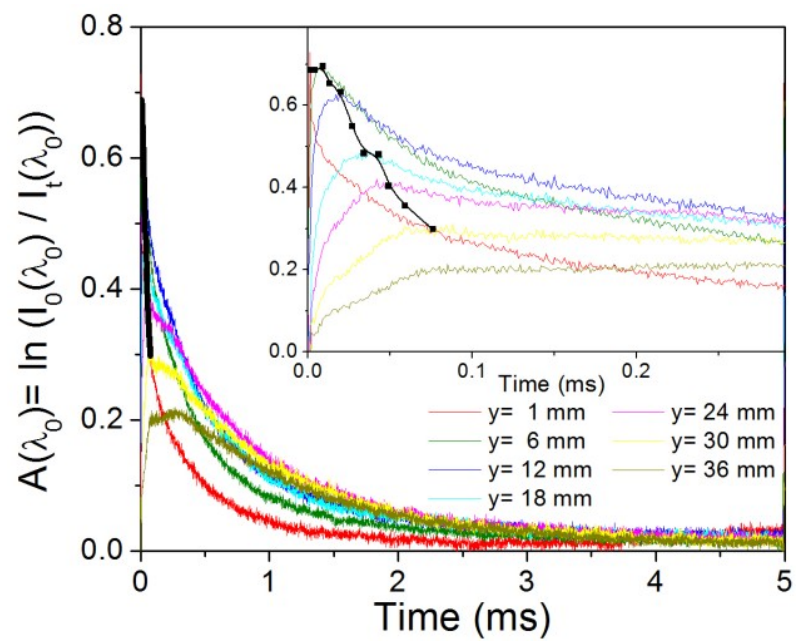

Fig. 9. Temporal evolution of maximum absorbance for aluminum spectral line at $394.4 \mathrm{~nm}$, for different distances from the target ( $p=20$ mTorr).



(a)

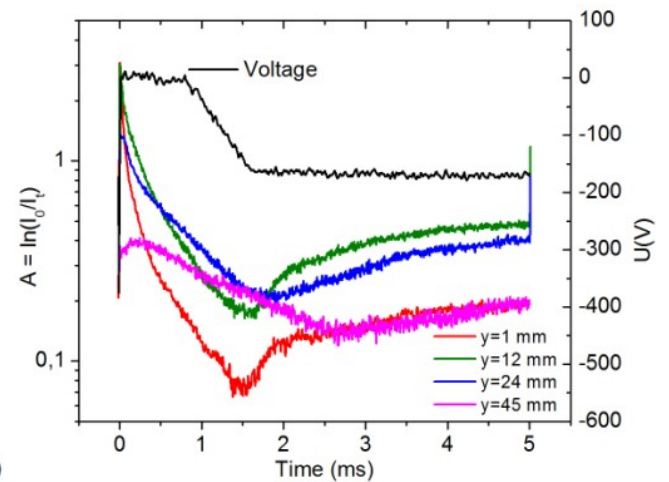

(b)

Fig. 10. (a) Time and space evolution of the density of argon metastable atom $\left(\mathrm{Ar}^{\mathrm{M}}\right)$ and (b) time evolution of maximum absorbance at $\lambda_{0}=811.531 \mathrm{~nm}$ for different distances from the target (aluminum target, $p$ $=20$ mTorr).

The second observation is that there are two different time periods in which kinetic of the argon metastable atoms is different. The first period corresponds to real afterglow regime which last $1 \mathrm{~ms}$ and follow immediately after high power pulse. During the high power pulse a large number of argon metastable atoms are produced mainly in front of the target where the electron trapping effect is maximum and electron-atom collision frequency is maximum. In afterglow regime the argon metastable atoms diffuses from high density plasma region in front of the target and their number decreases very rapidly in time close to the target mainly because of their collision with the metal surface (red curve in Fig.10b)[18], result proved also by the fact that with increasing distance from the target the decreasing rate in time of the 
metastable atoms number decreases. After about $1 \mathrm{~ms}$ the pre-ionization plasma is set-on in the system and almost steady number of metastable argon atoms are produced within the space between target and anode with non-uniform spatial distribution characteristic to magnetron discharge.

\section{Conclusion}

Diagnostic methods, based on laser diode absorption and fluorescence spectroscopy, have been adapted and used for investigation of the $2 \mathrm{D}$ spatial variation of the mean velocity distribution function of the metallic atoms sputtered form the target and the time and space evolution of the absorbing species density in a HiPIMS systems. The spatial characterization of $v d f$ of the metal atoms was performed in radial (parallel to the target) and axial (perpendicular to the target) direction of the discharge reactor for both DC and HiPIMS operating modes. For the same average discharge power, the obtained $v d f$ are comparable in HiPIMS (mean values) and DC. Each $v d f$ (axial and radial) exhibits two groups of atoms: one group of thermal atoms and one group of energetic atoms, showing larger velocity distribution in radial direction than in axial one. Because an important fraction of the sputtered atoms are ionized in HiPIMS, the energetic atoms population is lower than in DC mode.

The results show that the absorption profile in virtually any instant on the pulse period can be obtained. The absorption method has proved its efficiency for the buffer gas (argon atoms in metastable state) in HiPIMS systems and it can also be extended to the metallic sputtered species (aluminum or tungsten atoms). From the I-V characteristic of the HiPIMS mode results that the self sputtering process of the target metal is not dominant for short pulses (less than $10 \mu \mathrm{s}$ ).

\section{Acknowledgments}

This work was supported by the Romanian grant 540/2009 within the 'Idei' program.

\section{References}

[1] M. A. Lieberman and A. J. Lichtenberg, Principles of plasma discharges and materials processing, New York: John Wiley, 1994.

[2] B. N. Chapman, Glow discharge processes, New York: John Wiley, 1980.

[3] V. Kouznetsov, K. Macak, J. M. Schneider, U. Helmersson, I. Petrov, Surf. Coat. Technol. 122, 290-293 (1999). 
[4] K. Macak, V. Kouznetsov, J. Schneider, U. Helmersson, J. Vac. Sci. Technol. A 18,15331537 (2000).

[5] ITER Physics Basis, Nucl. Fusion 39, 2137 (1999).

[6] B. Unterberg, R. Jaspers, et al., Fusion Engineering and Design 86, 1797-1800 (2011).

[7] A. Anders, J. Andersson, A. Ehiasarian, J. Appl. Phys. 102, 113303 (2007).

[8] H. W. Müller, J. Adámek, et al., Nuclear Fusion 51(7), 073023 (2011).

[9] L. de Poucques, C. Vitelaru, T. M. Minea, J. Bretagne,G. Popa, Europhys. Lett. 82, 15002 (2008).

[10]C. Vitelaru, C. Aniculaesei, L. de Poucques, T. M. Minea, C. Boisse-Laporte, J. Bretagne, G Popa, J. Phys. D: Appl. Phys. 43, 124013 (2010).

[11]C. Vitelaru, V. Pohoata, C. Aniculaesei, V. Tiron, G. Popa, J. Appl. Phys. 109, 084911 (2011).

[12]C. Vitelaru, L. de Poucques, T. M. Minea, G. Popa, J. Appl. Phys. 109, 053307 (2011).

[13]M. Ganciu, M. Hecq, S. Konstantinidis, J. P. Dauchot, J. Bretagne, L. de Poucques, M. Touzeau, European Patent Appl. 4447072.2 (22 Mars 2004) WO 2005/090632.

[14]H. Scheibner, St. Franke, S. Soleyman, J. F. Behnke, C. Wilke, A. Dinklage, Rev. Sci. Instrum. 73, 378 (2002).

[15]M. Wolter, H. T. Do, S. Hartmut, R. Hippler, J. Phys. D: Appl. Phys. 38, 2390 (2005).

[16]J. Olejnicek, H. T. Do, Z. Hubicka, R. Hippler, L. Jastrabik, Jap. J. Appl. Phys., 45(10B), 8090 (2006).

[17]C. Vitelaru, T. Minea, L. de Poucques, M. Ganciu, G. Popa, Romanian J. Phys. 56, 47 (2011).

[18]P. Macko and N. Sadeghi, Plasma Sources Sci. Technol. 13, 303 (2004). 